\title{
Research on 3D Modeling of Point cloud data Based on Terrestrial Laser
}

\section{Scanner}

\author{
WU Meng ${ }^{1,3, a}$, WANG JianQiang ${ }^{1,2,3, b}$ and LUO $\operatorname{Han}^{1,3, c}$ \\ ${ }^{1}$ Faculty of Geomatics, East China Institute of Technology, 418 Guanglan Road, Nanchang, 330013, \\ China \\ ${ }^{2}$ Ditital Land Key Lab of Jiangxi Province, 418 Guanglan Road, Nanchang, 330013, China \\ ${ }^{3}$ Key laboratory of watershed ecology and geographical environment monitoring, National \\ Administration of Surveying, Mapping and Geoinformation, 418 Guanglan Road, Nanchang, \\ 330013, China \\ a493759991@qq.com, bwjq86991@163.com, '348847124@qq.com
}

Keywords: Terrestrial Laser Scanner; Modeling; Streamline point cloud

Abstract. 3D Laser Scanning Technology has been extensively studied because it can get large number of the target surface point cloud data quickly. In this paper, we use the terrestrial laser scanner to get the point cloud data of the rock surface, for these point cloud data, we discussed the effect of instrument scanning error and point clouds registration errors on the modeling of point cloud data, and propose methods to improve the point cloud processing according to the example, using the data processed by this method to establish a model of the rock surface. Experimental results show that the point cloud which processed by the improved method can effectively reduce the impact of point cloud data error on modeling.

\section{Introduction}

3D Laser Scanning Technology is a new mapping technology following the GPS technology that has become one of the important means to obtain spatial data [1]. Compared to traditional measurement, 3D Laser Scanner can continuous, automatic, rapid obtain the point cloud data of the surface of the target, has been widely used in various fields, including the protection of cultural relics, ancient buildings reconstructed, digital city, deformation monitoring, topographic survey, mine surveying etc [2,3].

Although the 3D laser scanner can obtain the high-density point cloud data, but if the whole point cloud are used to build the model, not only led to a long computer processing time, but also affect the smoothness of the model surface seriously due to the impact of the errors of the point cloud. Therefore, it is particularly important to process the point cloud data under the premise of ensuring the fineness of the point cloud [4]. In recent years, many people do a lot of researches to streamline the point cloud data, there are many specific ways to streamline the point cloud data, such as uniform grid method, plane fitting. This study is to reduce the impact of modeling caused by scanning and registration errors, we choose the algorithm of quadric surface fitting to streamline the point cloud data, this algorithm can get the ideal point cloud for modeling effectively.

\section{Data Acquisition}

We use the RIEGL VZ-400 laser scanner to acquire the data of point cloud, the scanning distance of this instrument up to 600 meters, scanning accuracy is $5 \mathrm{~mm}$ [5].

In order to reduce the amount of the point cloud data and get high-density point clouds, we scan 
360 degrees roughly for a short time, and then select the target of the rock areas for a long time scanning to obtain high-density point cloud data.

\section{Point cloud data preprocessing}

Point cloud data preprocessing includes point cloud data registration and de-noising. Point cloud registration is actually to find a rigid transformation from coordinate system 1 to coordinate system 2, so that the point cloud registration is to get the relationship of the rigid transformation [6]. The point cloud without registration shown in Figure 1, the instrument position of A1, A2 station overlapped in this Figure, the coordinates of the point cloud are in the coordinate system of the instrument itself.

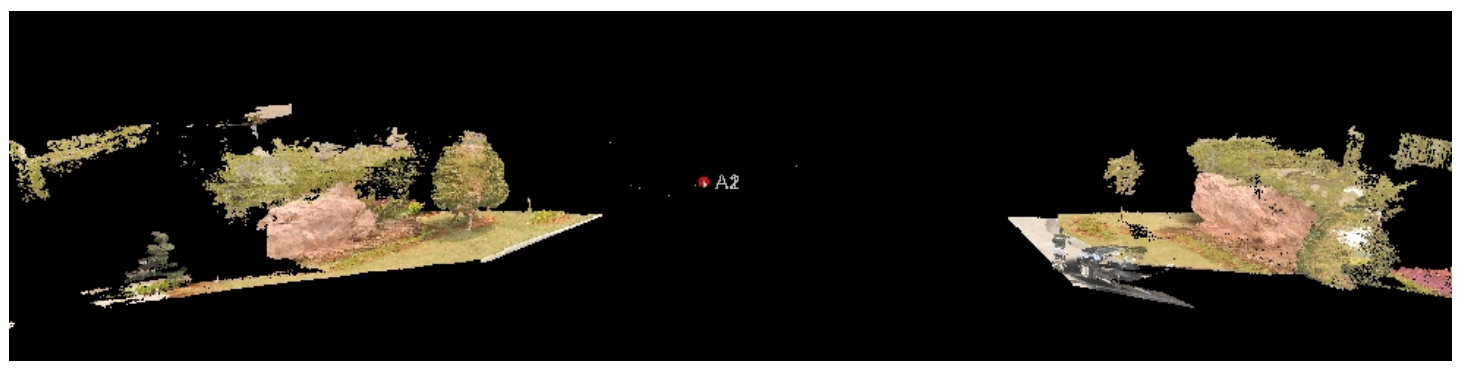

Fig. 1 The point cloud without registration

The coordinate of three-dimensional space can transformed by the seven parameter model, Assumed the coordinate of one point in the scanner's own coordinate system and the coordinate of the project are $\left(x^{\prime}, y^{\prime}, z^{\prime}\right)$ and $(x, y, z)$, the coordinate conversion can be achieved by translation and rotation, coordinate conversion formula can be expressed as

$$
\left(\begin{array}{l}
x \\
y \\
z \\
1
\end{array}\right)=\left(\begin{array}{cccc}
\cos \beta \cdot \cos \gamma & \cos \beta \cdot \sin \gamma & \sin \beta & t_{1} \\
-\cos \alpha \cdot \sin \gamma-\sin \alpha \cdot \sin \beta \cdot \cos \gamma & \cos \alpha \cdot \cos \gamma-\sin \alpha \cdot \sin \beta \cdot \sin \gamma & \sin \alpha \cdot \cos \beta & t_{2} \\
\sin \alpha \cdot \sin \gamma-\cos \alpha \cdot \sin \beta \cdot \cos \gamma & -\sin \alpha \cdot \cos \gamma-\cos \alpha \cdot \sin \beta \cdot \sin \gamma & \cos \alpha \cdot \cos \beta & t_{3} \\
0 & 0 & 0 & 1
\end{array}\right)\left(\begin{array}{l}
x^{\prime} \\
y^{\prime} \\
z^{\prime} \\
1
\end{array}\right)
$$

In this formula, $t_{i}$ are the 3 translation parameters, $\alpha, \beta, \gamma$ are the rotation angle of the scanner coordinate system around the $x, y, z$ axis of the project coordinate system.

The coordinates of the station and the target directly determines the size of $\alpha, \beta, \gamma$, therefore, the accuracy of the station and the target coordinates directly affect the accuracy of the data registration, but the coordinate of the station and the target must have errors, it will cause registration errors inevitably, the point cloud shown in Figure 2. 


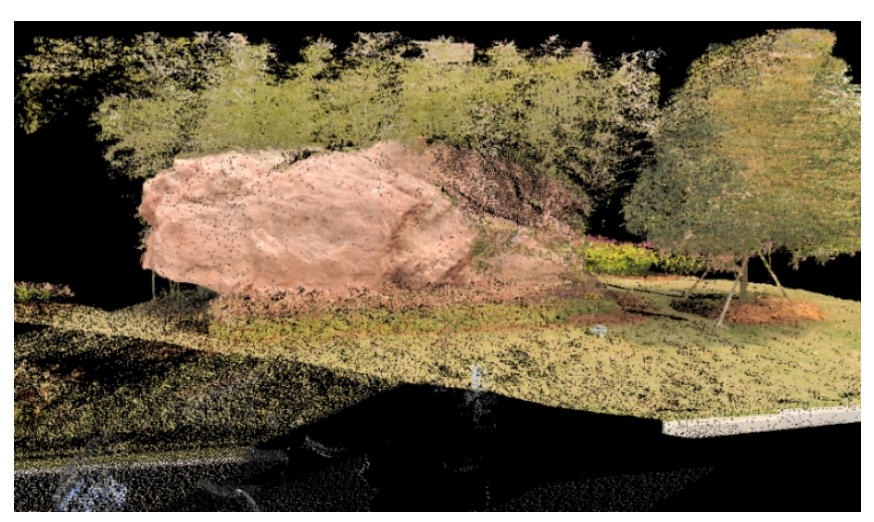

Fig.2 The registered point cloud

We de-noise the point cloud data when the registration of the point cloud data is completed. For the points which obvious deviation from the target, such as the next other buildings, trees, rocks, can be removed by hand directly. The point cloud generated by $3 \mathrm{D}$ laser scanner has more miscellaneous points there, which scattered around the body, especially the contour of the edge. For this points, usually delete them by hand or use the function of separation point or contour function to choice them and then delete them [7]. The de-noised point cloud data of the surface of the rock are shown in Figure 3, a total of 213,470 points.

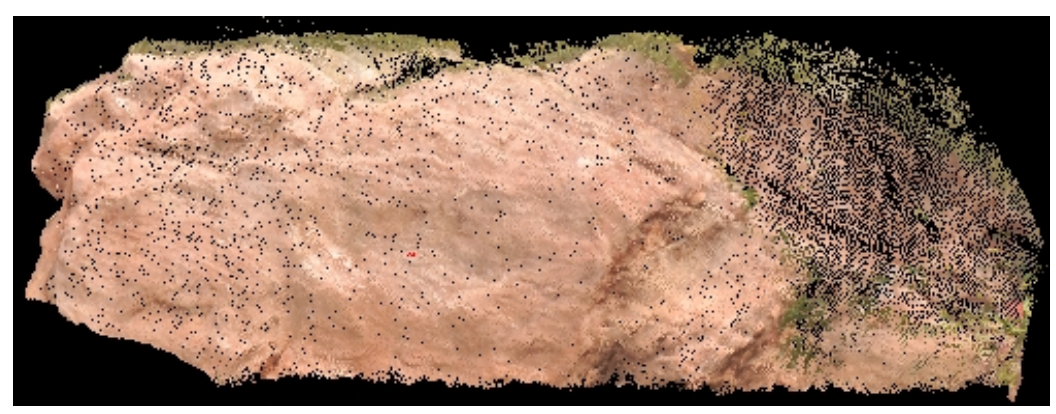

Fig.3 The de-noised point cloud data

\section{Point cloud data simplification and model reconstruction}

Due to the presence of the instrument and registration errors, point clouds are more or less deviation from the theoretical position, this way, the model reconstruction would have been affected. We model the de-noised point cloud directly, local model as shown in Figure 4. It is not difficult to see that, because of the influence of the scan error and registration error, the surface of the model is very rough, there are 419241 numbers of triangle nets in this model, and it takes 10 seconds to complete the modeling. 


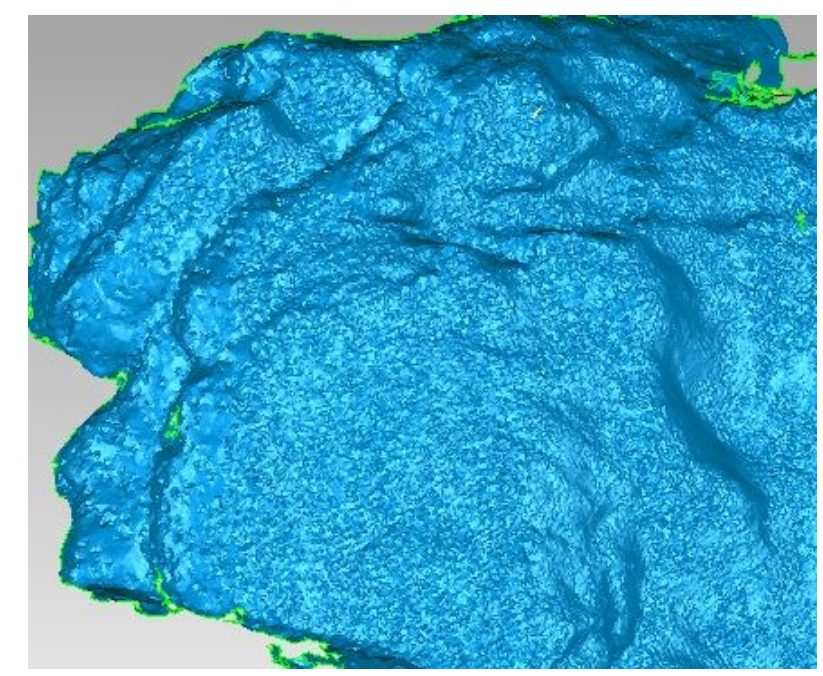

Fig. 4 Local model of the point cloud without streamlined

To make the surface of the model smoother under the premise of ensuring the model without losing texture features, we propose quadratic surface fitting to streamline the point cloud. This method according to the curvature of the point to streamline the point cloud data is simple and easy, it can retain the point cloud where the texture feature are big changes, and it streamline a lot of point cloud data where the texture feature little change.

\section{Point cloud segmentation}

There are no geometric relationship regulars between the original point cloud data, we need to separate the point cloud data in order to improve the speed of searching point data in the field, here we separate the point cloud data with the method of bounding box.

Using the method of bounding box to create big surrounding box surrounding the whole point cloud, we divide the large bounding box into a number of cubic lattice uniformly. Each cubic lattice adjacent with up to 26 other cube lattice, we find out $\mathrm{K}$ points which are the nearest neighboring point of the point $\mathrm{Pi}$ at the cube lattice which contains $\mathrm{Pi}$ and its 26 adjacent cube lattice, called neighborhood $\mathrm{m}$, using the least squares fitting quadric surface through these $\mathrm{K}$ points, as follows:

$$
Z(x, y)=a x^{2}+b x y+c y^{2}
$$

\section{Streamline the point cloud with quadratic surface fitting and model reconstruction}

We take the point cloud of a neighborhood $\mathrm{m}$, make the formula (3) take the minimum according to the principle of least squares.

$$
Q^{2}=\sum_{j}\left(a x_{j}^{2}+b x_{j} y_{j}+c y_{j}^{2}-z^{j}\right)^{2}, j \in(0, k)
$$

In this type: $x_{j}, y_{j}, z_{j}$ are the coordinates of the points in neighborhood, $\mathrm{k}$ is the number of points in the neighborhood. Derivative the coefficient of type (3) respectively and make its value is zero, then we get the following formula 


$$
\left\{\begin{array}{l}
\frac{\partial Q^{2}}{\partial a}=\sum_{j} 2 x_{j}^{2}\left(a x_{j}^{2}+b x_{j} y_{j}+c y_{j}^{2}-z_{j}\right)=0 \\
\frac{\partial Q^{2}}{\partial b}=\sum_{j} 2 x_{j} y_{j}\left(a x_{j}^{2}+b x_{j} y_{j}+c y_{j}^{2}-z_{j}\right)=0 \\
\frac{\partial Q^{2}}{\partial c}=\sum_{j} 2 y_{j}^{2}\left(a x_{j}^{2}+b x_{j} y_{j}+c y_{j}^{2}-z_{j}\right)=0
\end{array}\right.
$$

Simultaneous Equation (4) can solve the values of a, b, c.

According to the first basic formula of the surface

$$
(d s)^{2}=I=E(d x)^{2}+2 F d x d y+G(d y)^{2}
$$

And the second basic formula of the surface

$$
I I=-d r \cdot d n=L(d x)^{2}+2 M d x d y+N(d y)^{2}
$$

We can obtain the Gaussian curvature $\mathrm{K}$ and the local mean curvature $\mathrm{H}$ of all the points in the neighborhood, then calculates the overall mean curvature at all points. Through all over the point cloud, compared the local mean curvature and the average curvature of the point, we can determine the trade-off situation of the point. If the local mean curvature is less than the overall mean curvature $\mathrm{H}$ multiplied the threshold value a, that the distribution of the points in this area are relatively flat, then we just retain the point which Gaussian curvature $\mathrm{K}$ is maximum in this neighborhood; On the contrary, the distribution of the points in this area are relatively flat steep, then we just retain the points which Gaussian curvature K are larger than the local mean curvature.

In this algorithm, there are two quantities to restrict the results of the streamline: the side length of the cube lattice Ls and threshold value $a, L s=e^{*} K /(n+e p s)$, Where the e is the scaling factor, $K$ is the number of neighboring points. Keep threshold value $\mathrm{a}=4, \mathrm{~K}=10$ unchanged, we take a different scale factor e, the streamlined point cloud shown in Figure 5, the local model of the streamlined point cloud shown in Figure 6, the specific result of streamline shown in Table 1. When $\mathrm{e}=4$, the feature point well kept, but the point cloud distributed unevenly, too much in some areas, the surface roughness model are some improvements when compared with the untreated, the modeling efficiency are Significantly enhanced. When $\mathrm{e}=12$, the feature point well kept, the distribution of point cloud is appropriate, the contour curve are complete, the surface roughness model is a significant improvement when compared with the untreated, the amount of the model data are minimal, and the most effective it is.

Table. 1 The result of streamline

\begin{tabular}{cccccc}
\hline e & Ls[mm] & $\begin{array}{c}\text { the number } \\
\text { of } \\
\text { Streamlined } \\
\text { point cloud }\end{array}$ & $\begin{array}{c}\text { Streamline } \\
\text { rate }\end{array}$ & $\begin{array}{c}\text { The } \\
\text { number of } \\
\text { triangle } \\
\text { nets }\end{array}$ & $\begin{array}{c}\text { Modeling } \\
\text { time[s] }\end{array}$ \\
\hline 4 & 7.5 & 111867 & $52.40 \%$ & 219278 & 5 \\
12 & 22.4 & 50482 & $23.65 \%$ & 99065 & 2 \\
\hline
\end{tabular}




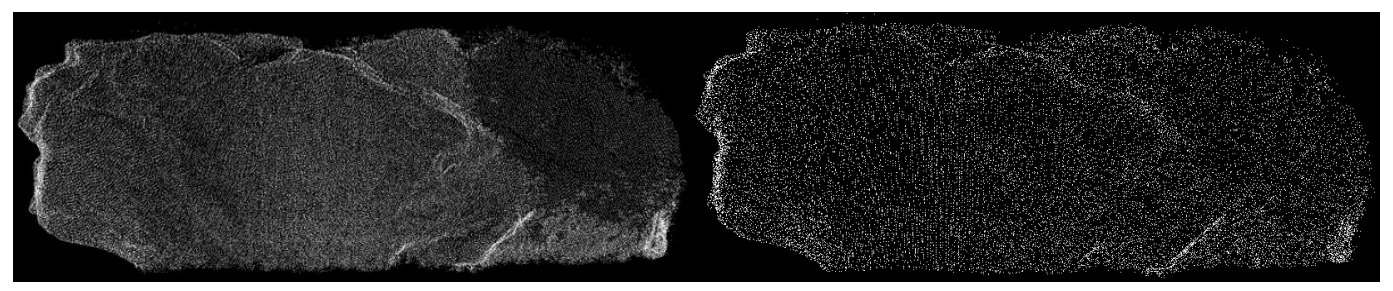
(a) $\mathrm{e}=4$
(b) $\mathrm{e}=12$

Fig. 5 The streamlined point cloud

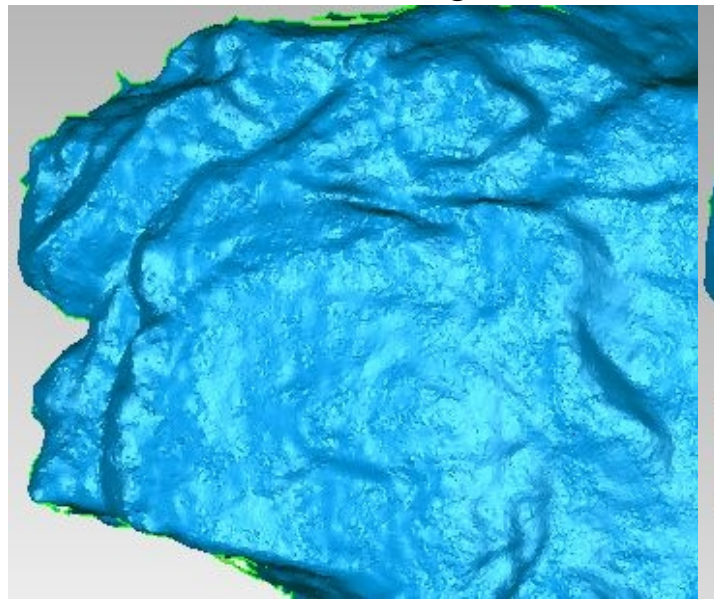

(a) $\mathrm{e}=4$

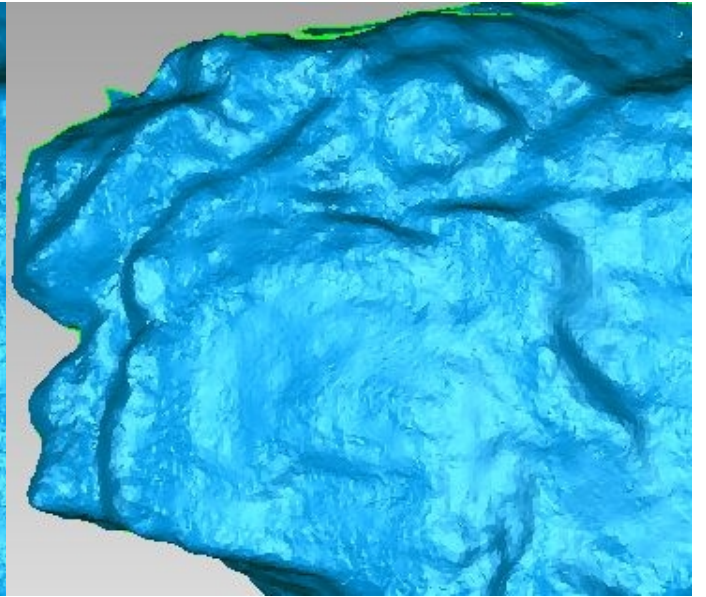

(b) $\mathrm{e}=12$

Fig. 6 Local model of the point cloud with streamlined

Finally, we use the camera to shoot HD picture of the rock surface, using Photoshop to make the textures, then import the streamlined model to 3DMAX and texture it. The final result of the model shown in Figure 7, the texture of the model is clear, and the performance the details outstanding strength.

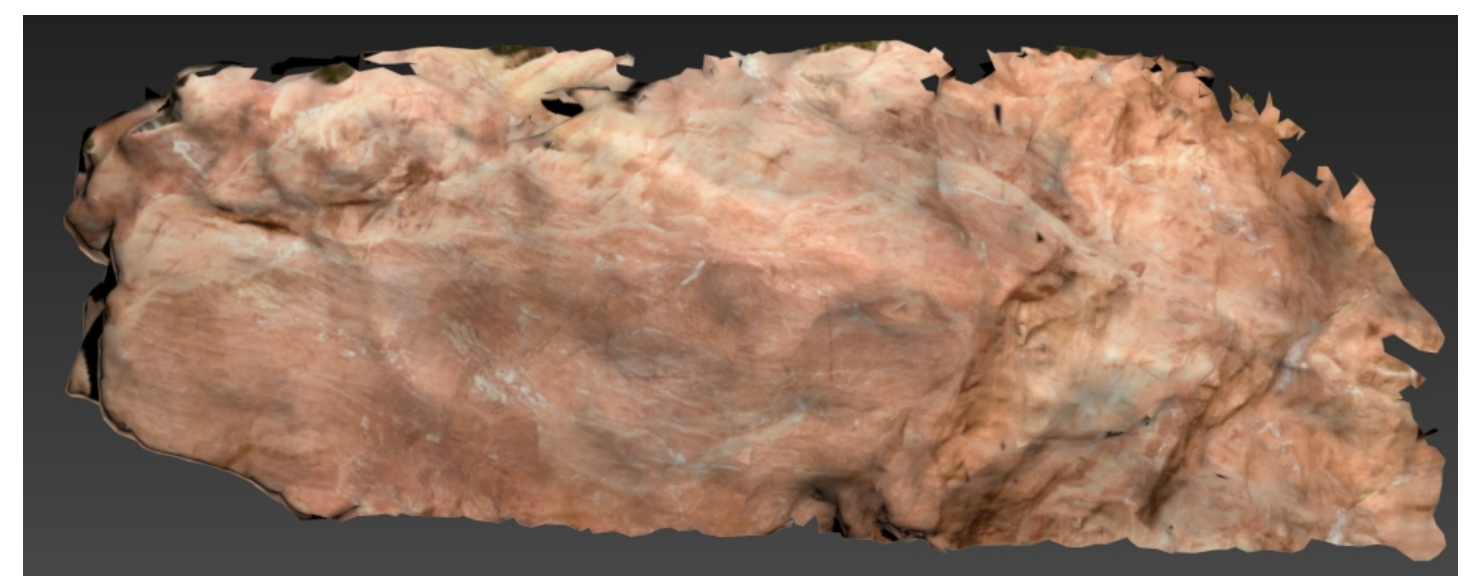

Fig.7 The textured model

\section{Conclusion}

We use the terrestrial laser scanner to get the point cloud data of the rock surface, and apply the algorithm of quadric surface fitting to streamline the point cloud data, From the streamlined result we can see that this algorithm can effectively get the ideal point cloud for modeling, especially for the data which the curvature changes larger and contains more features, and improved the smoothness of the surface of the model under the premise of ensuring the model without losing texture features. But this article is just research on modeling one side of rock, for more complex 
objects, such as a large building modeling, the next step of this article is to research this.

\section{Acknowledgements}

This work was financially supported by the Jiangxi Provincial Key Laboratory of Digital Land Foundation (DLLJ201307), National Natural Science Foundation of China (41304020) and East China Institute of Technology Dr. Scientific Research Foundation (DHBK201114).

\section{References}

[1] LIU Zhong-xi, CHEN Kai. Review the ground 3D laser scanner matching technology[J].Shanxi Architecture, 2013, 39(5): 205-207.

[2] ZHANG Hui-xia, CHEN Yin-jin, LIU Guo-bo. Campus building modelling based on3D laser scanning[J]. Engineering Of Surveying And Mapping, 2010, 19(1): 32-34.

[3] Zhang Y, Chen H, Du Z Q. 3D Modeling of Underground Geological Park Based on Terrestrial Laser Scanner[J]. Advanced Materials Research, 2013, 748: 1119-1124.

[4] LIU Tao, XU Zheng, SHA Cheng-mei, ZHAO Jun-tian. Curvature Estimation of Scattered-point Cloud Data Based on Bounding Box Method[J]. Science Technology and Engineering, 2009, (12):3333-3336.

[5] Zang Ke. The Research of Scanning Data Based on Riegl3D-Laser Scanner[J]. Journal of Capital Normal University: Natural Science Edition, 2007,(1):77-82.

[6] Zhao Xu. Research on 3D Reconstruction of Point Cloud from Terrestrial Laser Scanning[D]. WuHan University, 2010:35-36.

[7] Liu Xiuguo, Wang Hongping, Yu Mingxu. 3D Model Reconstruction Based on Laser Scanning Technique[J]. Laser \& Optoelectronics Progress, 2011,48(8):112-117. 